\title{
Sleep Stage Influence on the Autonomic Modulation of Sleep Apnea Syndrome
}

\author{
Mireia Calvo ${ }^{1}$, Raimon Jané $e^{1,2,3}$ \\ ${ }^{1}$ Institute for Bioengineering of Catalonia (IBEC), the Barcelona Institute of Science and Technology \\ (BIST), Barcelona, Spain \\ ${ }^{2}$ Universitat Politècnica de Catalunya (UPC)-Barcelona Tech, Barcelona, Spain \\ ${ }^{3}$ Biomedical Research Networking Centre in Bioengineering, Biomaterials and Nanomedicine \\ (CIBER-BBN), Barcelona, Spain
}

\begin{abstract}
Hypoxia induced by obstructive sleep apnea (OSA) leads to the deregulation of the autonomic nervous system (ANS), resulting in an abnormally increased sympathetic activity. Since ANS modulation varies throughout the night, notably for each sleep stage, the hypnogram and heart rate signals of 81 OSA patients were collected during a polysomnography. They were classified as mild-moderate $(n=44)$ or severe $(n=37)$ based on their apnea-hypopnea index (AHI). Spectral heart rate variability $(H R V)$ series were extracted by a time-frequency approach. These series were then averaged for each sleep stage, in order to compare the sympathetic modulation of mild-moderate and severe patients at the following phases: rapid eye movement (REM), S1, S2 and SWS (slow wave sleep). According to normalized power at the lowfrequency band $\left(L F_{n u}\right)$ values, severe OSA seems to be associated with an increased sympathetic modulation at non-REM sleep. Moreover, a decreased autonomic variability throughout the night may be related to a reduced adaptability of the cardiovascular system, characterizing a more advanced stage of the disease. These results provide further evidence for the role of autonomic alterations induced by hypoxia, suggesting the use of HRV analysis, together with AHI, for the study of OSA severity.
\end{abstract}

\section{Introduction}

Obstructive events in sleep apnea syndrome cause arterial oxygen desaturation, hypercapnia, intrathoracic pressure alterations and, frequently, sleep disruption. The negative intrathoracic pressures generated during obstructed inspiratory efforts induce transient reductions in the left ventricular stroke volume, heart rate and systemic arterial pressure. As a result, the immediate postapnea period is characterized by a notable, transient surge in systemic arterial pressure caused by a sympathetic nervous system ac- tivation [1]. Indeed, studies using supplemental oxygen have shown that carotid chemoreceptor stimulation by asphyxia seems to be the most important cause of apneainduced sympathetic activation and blood pressure elevation $[2,3]$.

Accordingly, several studies have reported that sympathetic modulation is increased in obstructive sleep apnea (OSA) patients during sleep and wakefulness $[4,5]$ and reductions in muscle sympathetic nerve activity have been documented following continuous positive airway pressure (CPAP) treatment $[6,7]$, indicating a causal relationship between OSA and sympathetic nervous system activation and thus suggesting sympathetic modulation as an indicator of OSA severity.

However, the level of sympathetic activation induced by apnea seems to be influenced by several factors. Sleep disruption increases sympathetic activity [8] and respiratory arousals seem to boost the effects [9]. For their part, cortical arousals probability of occurence after obstructive events have been reported to depend on sleep stage [10]. Slow wave sleep (SWS) has been defined as a "safety state" preventing breathing abnormalities since it has shown a lower number of shorter obstructive events and arousals, as well as less severe desaturations $[10,11]$. In contrast, obstructive events seem to be more frequent [12-14], of longer duration $[15,16]$ and associated with more severe desaturations $[17,18]$ during rapid eye movement (REM) sleep. In fact, previous studies on healthy subjects confirm that the level of sympathetic activation depends on sleep stage $[19,20]$. Thus, autonomic assessment so as to analyze the potential of sympathetic modulation as an indicator of OSA severity should take into account this information.

Therefore, this study analyzes the sympathetic modulation at night of 81 OSA patients at different levels of severity. Apart from a whole-night analysis, sympathetic modulation was also characterized for each sleep stage. The comparison of mild-moderate and severe OSA patients 
could provide new insights in the understanding of the disease, suggesting the use of heart rate variability (HRV) analysis, together with apnea-hypopnea index (AHI), for the study of OSA severity.

\section{Materials and methods}

\subsection{Study population}

The hypnogram and heart rate signals of 81 patients suffering from OSA were collected during a standard polysomnography (Minisomno; Sefam, Nancy, France) at the University Hospital Germans Trias i Pujol in Badalona, Spain (2008-2009) [21]. The study protocol was approved by the hospital ethics committee and all patients provided their written informed consent to participate in the study.

Participant ages ranged from 23 to 75 years old (51.4 \pm 11.0 years old $)$ and $68(83.9 \%)$ were males. They were classified as mild-moderate $(n=44)$ or severe $(n=37)$ based on their AHI (total apneas and hypopneas per sleep hour), by setting the cut-off point at 30 . All participants were free of any upper airway infection and other diseases throughout the study and none had undergone treatment for snoring or were taking any medication at the time of data collection. Table 1 summarizes the main clinical characteristics of both study groups, showing a higher proportion of men, slightly older and with greater body mass index (BMI) values in the severe OSA group.

Table 1. Clinical characteristics of OSA patients. BMI: Body Mass Index; AHI: Apnea-Hypopnea Index.

\begin{tabular}{lcc}
\hline \hline & $\begin{array}{c}\text { Mild-moderate } \\
(\mathrm{n}=44)\end{array}$ & $\begin{array}{c}\text { Severe } \\
(\mathrm{n}=37)\end{array}$ \\
\hline Age, years old & $50.4 \pm 11.9$ & $52.6 \pm 9.8$ \\
Male sex, $n(\%)$ & $35(79.6 \%)$ & $33(89.2 \%)$ \\
BMI, $\mathrm{kg} / \mathrm{m}^{2}$ & $27.5 \pm 3.3$ & $30.8 \pm 4.6$ \\
AHI, $h^{-1}$ & $21.6 \pm 9.7$ & $68.2 \pm 20.5$ \\
\hline \hline
\end{tabular}

\subsection{Heart rate variability analysis}

In order to remove low-frequency noise, heart rate signals were high-pass filtered at $0.03 \mathrm{~Hz}$ with a $4^{\text {th }}$ order Butterworth filter applied in both forward and backward directions so as to remove phase distortion. Then, spectral non-stationary heart rate variability (HRV) series were extracted by means of a Smoothed Pseudo Wigner-Ville distribution (SPWVD) approach. This quadratic timefrequency method, defined as the Fourier transform of the instantaneous autocorrelation function, was applied with a smoothing kernel that attenuates interferences while maintaining an acceptable time-frequency resolution. As in
[22], kernel parameters were adjusted to obtain temporal and spectral resolutions of 16.7 seconds and $0.033 \mathrm{~Hz}$, respectively.

Then, HRV was measured as the total power of SPWVD in the low-frequency (LF) and high-frequency (HF) bands. Assuming that sympathetic and parasympathetic activites always lie within the standard LF and HF bands at night, they were respectively fixed to $0.04-0.15 \mathrm{~Hz}$ and $0.15-0.4 \mathrm{~Hz}$, for the whole night. Finally, these time series accounting for the sympathetic and parasympathetic influences of the autonomic nervous system on heart rate, were normalized and expressed as percentages of the total power, defined as the sum of both spectral bands $(T P(t)=L F(t)+H F(t))$, leading to the time series $L F_{n u}(t)$ and $H F_{n u}(t)$ :

$$
\begin{aligned}
L F_{n u}(t) & =\frac{L F(t)}{T P(t)} \cdot 100 \\
H F_{n u}(t) & =\frac{H F(t)}{T P(t)} \cdot 100
\end{aligned}
$$

From this definition of normalization, it should be noted that $L F_{n u}(t)=100-H F_{n u}(t)$ and, thus, statistical results for both time series are identical. Since the main objective of this study was to characterize the sympathetic modulation of OSA patients at night, the following sections mainly show and discuss the results obtained for $L F_{n u}(t)$.

Apart from comparing the whole-night average sympathetic modulation $\left(m L F_{n u}\right)$ and its variability, based on the standard deviation $\left(s L F_{n u}\right)$, between mild-moderate and severe patients, $L F_{n u}(t)$ series were also averaged for each sleep stage, in order to compare study groups at the following sleep phases: S1, S2, SWS (slow wave sleep) and REM (rapid eye movement).

Statistical analyses were performed by means of MannWhitney U non-parametric tests for the comparison of mild-moderate and severe patients and by Kruskal-Wallis tests for the study of differences among sleep stages within each study group.

\section{Results}

Figure 1 illustrates an example of the SPWVD obtained for a severe OSA patient at night. The graph represents those frequencies composing LF and HF bands, delimited by a horizontal dashed white line. In the second panel, the $L F(t)$ and $H F(t)$ time series, obtained from the total power at these bands, are displayed. In the third panel, the acquired $L F_{n u}(t)$ series can be observed, showing relatively high values.

Figure 2 displays the boxplots corresponding to the mean and standard deviation values of $L F_{n u}$ for the whole 

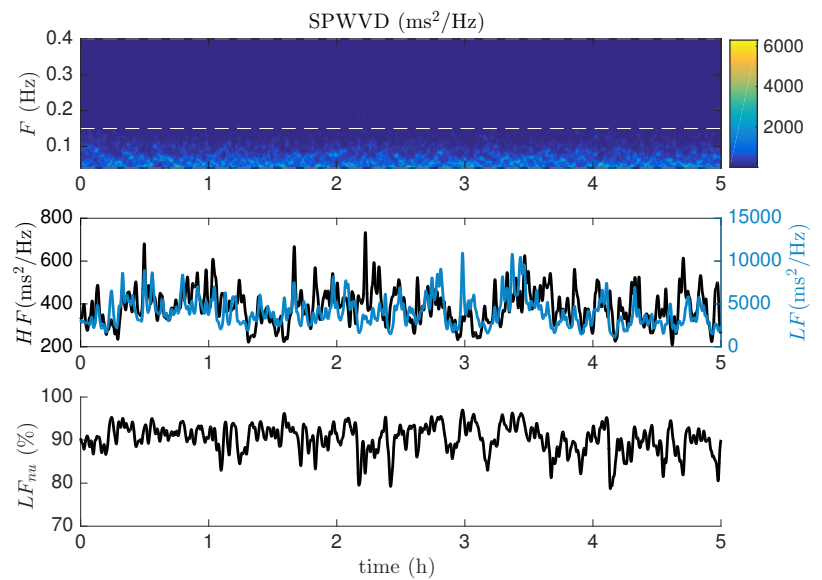

Figure 1. SPWVD and HRV time series for a severe OSA patient $\left(\mathrm{AHI}=42 \mathrm{~h}^{-1}\right)$. In the first panel, $\mathrm{LF}(0.04-0.15 \mathrm{~Hz})$ and $\mathrm{HF}(0.15-0.4 \mathrm{~Hz})$ bands are respectively represented below and above the horizontal dashed white line.

night ( $m L F_{n u}$ and $\left.s L F_{n u}\right)$, showing statistically significant differences between mild-moderate $(\mathrm{M})$ and severe (S) patients ( $p$-values $<0.01$ ). More specifically, the results indicate that severity seems to be associated with a higher global sympathetic activation and with a lower variability throughout the night.
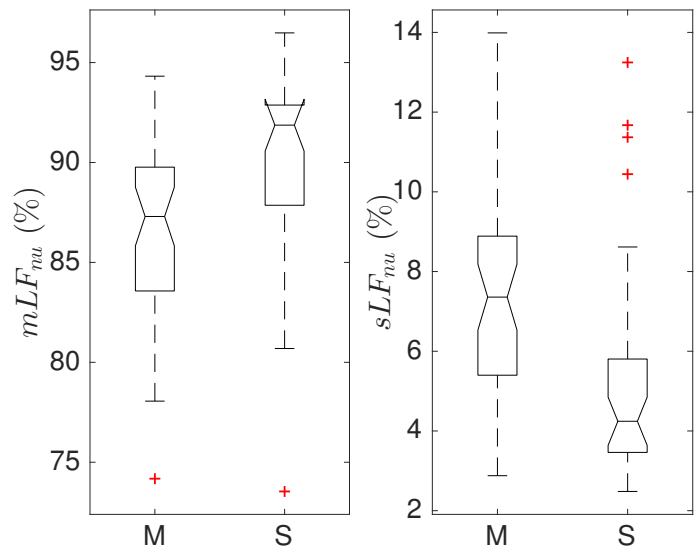

Figure 2. Boxplots for the whole-night $m L F_{n u}$ and $s L F_{n u}$, in mild-moderate (M) and severe (S) patients. In both statistical comparisons, $p$-value $<0.001$.

Finally, Figure 3 and Table 2 summarize the results obtained for sleep-stage analysis, showing statistically significant differences in non-REM sleep between mildmoderate and severe OSA patients.

Severe patients showed higher $m L F_{n u}$ values for nonREM stages (S1: $p=0.004$; S2: $p<0.001$; SWS: $p<0.001$ ), while no significant differences between

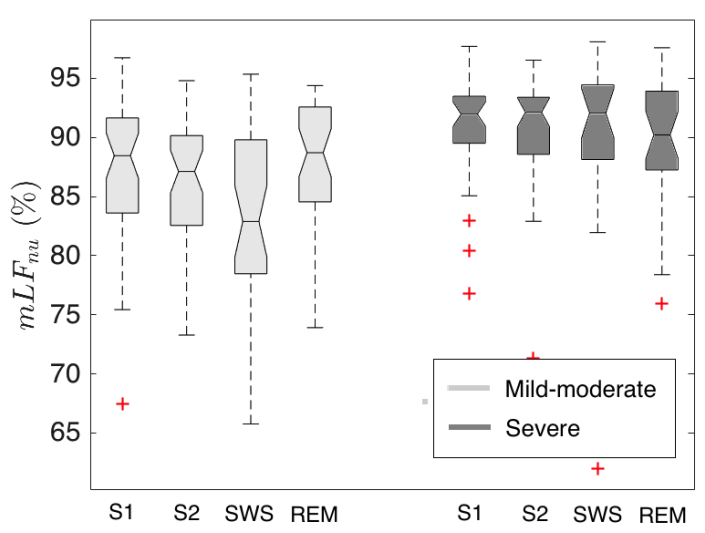

Figure 3. Boxplots for the $m L F_{n u}$ of each sleep stage, in mild-moderate and severe OSA patients.

Table 2. Average $L F_{n u}$ results for sleep-stage analysis (mean \pm standard deviation) in mild-moderate and severe OSA patients. ${ }^{*} p$-value $<0.05$, based on Mann-Whitney test.

\begin{tabular}{lccc}
\hline \hline Sleep stage & $\begin{array}{c}\text { Mild-moderate } \\
(\mathrm{n}=44)\end{array}$ & $\begin{array}{c}\text { Severe } \\
(\mathrm{n}=37)\end{array}$ & p-value \\
\hline S1 & $87.5 \pm 5.8$ & $90.7 \pm 4.2$ & $0.004^{*}$ \\
S2 & $86.5 \pm 5.0$ & $90.4 \pm 4.7$ & $<0.001^{*}$ \\
SWS & $83.0 \pm 7.9$ & $89.1 \pm 9.1$ & $<0.001^{*}$ \\
REM & $88.0 \pm 5.0$ & $89.8 \pm 5.3$ & 0.059 \\
\hline \hline
\end{tabular}

groups were observed at REM sleep ( $p=0.059$ ).

Indeed, while patients with mild-moderate OSA showed significant differences among sleep stages $(p=0.014)$, decreasing sympathetic activation with deeper sleep, severe patients showed a similar sympathetic modulation throughout the night $(p=0.978)$.

\section{Conclusions}

This study analyzes and compares the sympathetic modulation at night of 81 OSA patients at different levels of severity. Regarding whole-night analysis, severe OSA seems to be associated with an increased sympathetic activation. Moreover, a decreased autonomic variability throughout the night may be related to a reduced adaptability of the cardiovascular system, characterizing a more advanced stage of the disease.

Sympathetic modulation was also characterized for each sleep stage. While in mild-moderate patients sympathetic modulation seems to decrease with deeper sleep stages and significantly increase during REM sleep, a similarly elevated sympathetic activation across all sleep stages, in- 
cluding REM sleep, is observed in severe OSA patients. Thus, when comparing both groups, in more severe patients, a higher sympathetic modulation is observed during non-REM sleep stages, when a predominant vagal activation is expected. In contrast, no statistically significant differences were observed during REM sleep, when levels of sympathetic control have been reported to be even higher than in wakefulness.

Although other factors, such as age or gender, could also affect sleep apnea conditions [23, 24], these results show the potential of autonomic assessment throughout sleep stages for the study of OSA severity.

\section{References}

[1] H. Schneider, et al., "Neural and local effects of hypoxia on cardiovascular responses to obstructive apnea," J Appl Physiol, vol. 88, pp. 1093-1102, 2000.

[2] U. Leuenberger, et al., "Surges of muscle sympathetic nerve activity during obstructive apnea are linked to hypoxemia," J Appl Physiol, vol. 79, pp. 581-588, 1995.

[3] B. J. Morgan, et al., "Neurocirculatory consequences of negative intrathoracic pressure vs. asphyxia during voluntary apnea," J Appl Physiol, vol. 74, pp. 2969-2975, 1993.

[4] J. T. Carlson, et al., "Augmented resting sympathetic activity in awake patients with obstructive sleep apnea," Chest, vol. 103, pp. 1763-1768, 1993.

[5] K. Narkiewicz, et al., "Contribution of tonic chemoreflex activation to sympathetic activity and blood pressure in patients with obstructive sleep apnea," Circulation, vol. 97, pp. 943-945, 1998.

[6] V. A. Imadojemu, et al., "Sympathetic chemoreflex responses in obstructive sleep apnea and effects of continuous positive airway pressure therapy," Chest, vol. 131, pp. 1406-1413, 2007.

[7] K. Narkiewicz, et al., "Nocturnal continuous positive airway pressure decreases daytime sympathetic traffic in obstructive sleep apnea," Circulation, vol. 100, pp. 23322335, 1999.

[8] B. J. Morgan, et al., "Neurocirculatory consequences of abrupt change in sleep state in humans," J Appl Physiol, vol. 80, pp. 1627-1636, 1996.

[9] B. J. Morgan, et al., "Blood pressure perturbations caused by subclinical sleep-disordered breathing," Sleep, vol. 21, pp. 737-746, 1998.

[10] T. Leppannen, et al., "Differences in arousal probability and duration after apnea and hypopnea events in adult OSA patients," Physiol Meas, vol. 10, no. 7, pp. 821-824, 2008.

[11] A. Turoff, et al., "Sleep duration and quality in heart failure patients," Sleep Breath, vol. 21, pp. 919 - 927, 2017.

[12] F. Campos-Rodriguez, et al., "Clinical and polysomnographic features of rapid-eye-movement-specific sleepdisordered breathing," Arch Bronconeumol, vol. 45, pp. 330-334, 2009.

[13] A. Sunnetcioglu, et al., "Obstructive sleep apnea related to rapid-eye-movement or non-rapid-eye-movement sleep: comparison of demographic, anthropometric, and polysomnographic features," J Bras Pneumol, vol. 42, pp. 48-54, 2016.

[14] N. M. Punjabi, et al., "The association between daytime sleepiness and sleep-disordered breathing in NREM and REM sleep," in Sleep, 2002.

[15] B. B. Koo and W. Mansour, "Correlates of obstructive apnea duration," Lung, vol. 192, pp. 185-190, 2014.

[16] F. Siddiqui, et al., "Half of patients with obstructive sleep apnea have a higher NREM AHI than REM AHI," Sleep Med, vol. 7, pp. 281-285, 2006.

[17] F. Series, et al., "Influence of apnea type and sleep stage on nocturnal postapneic desaturation," Am Rev Respir Dis, vol. 141, pp. 1522-1526, 1990.

[18] L. J. Findley, et al., "Apnea duration and hypoxemia during REM sleep in patients with obstructive sleep apnea," Chest, vol. 87, pp. 432-436, 1985.

[19] S. Elsenbruch, et al., "Heart rate variability during waking and sleep in healthy males and females," in Sleep, vol. 22, pp. 1067-1071, 1999.

[20] M. P. Villa, et al., "Effects of sleep stage and age on shortterm heart rate variability during sleep in healthy infants and children," Chest, vol. 117, pp. 460-466, 2000.

[21] J. A. Fiz, et al., "Continuous analysis and monitoring of snores and their relationship to the apneahypopnea index," The Laryngoscope, vol. 120, no. 4, pp. 854-862, 2010.

[22] M. Calvo, et al., "Multivariate classification of Brugada syndrome patients based on autonomic response to exercise testing," PloS one, vol. 13, no. 5, pp. e0197367, 2018.

[23] T. Leppannen, et al., "Severity of individual obstruction events increases with age in patients with obstructive sleep apnea," Sleep Med, vol. 37, pp. 32-37, 2017.

[24] T. Leppannen, et al., "Severity of individual obstruction events is gender dependent in sleep apnea," Sleep Breath, vol. 21, pp. 397-404, 2017.

\section{Acknowledgments}

This work was funded by the European Union's Horizon 2020 research and innovation programme under the Marie Sklodowska-Curie grant agreement no. 712754, the Spanish Ministry of Economy and Competitiveness under the Severo Ochoa grant SEV-20140425 (2015-2019), the CERCA Programme, the Secretaria d'Universitats i Recerca del Departament d'Empresa i Coneixement de la Generalitat de Catalunya (GRC 2017 SGR 01770), the Spanish Ministry of Economy and Competitiveness (DPI2015-68820-R MINECO/FEDER) and by MCIU/AEI/FEDER (RTI2018-098472-B-I00).

Address for correspondence:

Raimon Jané

Institute for Bioengineering of Catalonia (IBEC)

Campus Diagonal-Besòs UPC, edifici C, 5a planta

Av. d'Eduard Maristany, 16, 08019, Barcelona, Spain

rjane@ibecbarcelona.eu 